\title{
Van der Waerden/Schrijver-Valiant like Conjectures and Stable (aka Hyperbolic) Homogeneous Polynomials: One Theorem for all
}

\author{
Leonid Gurvits \\ Los Alamos National Laboratory \\ gurvits@lanl.gov \\ Submitted: Jul 29, 2007; Accepted: Apr 29, 2008; Published: May 5, 2008 \\ Mathematics Subject Classification: 05E99
}

\begin{abstract}
Let $p$ be a homogeneous polynomial of degree $n$ in $n$ variables, $p\left(z_{1}, \ldots, z_{n}\right)=$ $p(Z), Z \in C^{n}$. We call such a polynomial $p$ H-Stable if $p\left(z_{1}, \ldots, z_{n}\right) \neq 0$ provided the real parts $\operatorname{Re}\left(z_{i}\right)>0,1 \leq i \leq n$. This notion from Control Theory is closely related to the notion of Hyperbolicity used intensively in the PDE theory.

The main theorem in this paper states that if $p\left(x_{1}, \ldots, x_{n}\right)$ is a homogeneous H-Stable polynomial of degree $n$ with nonnegative coefficients; $\operatorname{deg}_{p}(i)$ is the maximum degree of the variable $x_{i}, C_{i}=\min \left(\operatorname{deg}_{p}(i), i\right)$ and

$$
\operatorname{Cap}(p)=\inf _{x_{i}>0,1 \leq i \leq n} \frac{p\left(x_{1}, \ldots, x_{n}\right)}{x_{1} \cdots x_{n}}
$$

then the following inequality holds

$$
\frac{\partial^{n}}{\partial x_{1} \ldots \partial x_{n}} p(0, \ldots, 0) \geq C a p(p) \prod_{2 \leq i \leq n}\left(\frac{C_{i}-1}{C_{i}}\right)^{C_{i}-1}
$$

This inequality is a vast (and unifying) generalization of the Van der Waerden conjecture on the permanents of doubly stochastic matrices as well as the SchrijverValiant conjecture on the number of perfect matchings in $k$-regular bipartite graphs. These two famous results correspond to the $\mathbf{H}$-Stable polynomials which are products of linear forms.

Our proof is relatively simple and "noncomputational"; it uses just very basic properties of complex numbers and the AM/GM inequality.
\end{abstract}




\section{The permanent, the mixed discriminant, the Van Der Waerden conjecture(s) and homogeneous poly- nomials}

Recall that an $n \times n$ matrix $A$ is called doubly stochastic if it is nonnegative entry-wise and its every column and row sum to one. The set of $n \times n$ doubly stochastic matrices is denoted by $\Omega_{n}$. Let $\Lambda(k, n)$ denote the set of $n \times n$ matrices with nonnegative integer entries and row and column sums all equal to $k$. We define the following subset of rational doubly stochastic matrices: $\Omega_{k, n}=\left\{k^{-1} A: A \in \Lambda(k, n)\right\}$. In a 1989 paper [2] R.B. Bapat defined the set $D_{n}$ of doubly stochastic $n$-tuples of $n \times n$ matrices.

An $n$-tuple $\mathbf{A}=\left(A_{1}, \ldots, A_{n}\right)$ belongs to $D_{n}$ iff $A_{i} \succeq 0$, i.e. $A_{i}$ is a positive semi-definite matrix, $1 \leq i \leq n ; \operatorname{tr} A_{i}=1$ for $1 \leq i \leq n ; \sum_{i=1}^{n} A_{i}=I$, where $I$, as usual, stands for the identity matrix. Recall that the permanent of a square matrix $\mathrm{A}$ is defined by

$$
\operatorname{per}(A)=\sum_{\sigma \in S_{n}} \prod_{i=1}^{n} A(i, \sigma(i)) .
$$

Let us consider an $n$-tuple $\mathbf{A}=\left(A_{1}, A_{2}, \ldots A_{n}\right)$, where $A_{i}=\left(A_{i}(k, l): 1 \leq k, l \leq n\right)$ is a complex $n \times n$ matrix $(1 \leq i \leq n)$. Then

$$
\operatorname{Det}_{\mathbf{A}}\left(t_{1}, \ldots, t_{n}\right)=\operatorname{det}\left(\sum_{1 \leq i \leq n} t_{i} A_{i}\right)
$$

is a homogeneous polynomial of degree $n$ in $t_{1}, t_{2}, \ldots, t_{n}$. The number

$$
D(\mathbf{A}):=D\left(A_{1}, A_{2}, \ldots, A_{n}\right)=\frac{\partial^{n}}{\partial t_{1} \cdots \partial t_{n}} \operatorname{Det}_{\mathbf{A}}(0, \ldots, 0)
$$

is called the mixed discriminant of $A_{1}, A_{2}, \ldots, A_{n}$.

The mixed discriminant is just another name, introduced by A.D. Alexandrov, for 3dimensional Pascal's hyperdeterminant. The permanent is a particular (diagonal) case of the mixed discriminant. I.e. define the following homogeneous polynomial

$$
\operatorname{Prod}_{A}\left(t_{1}, \ldots, t_{n}\right)=\prod_{1 \leq i \leq n} \sum_{1 \leq j \leq n} A(i, j) t_{j}
$$

Then the next identity holds:

$$
\operatorname{per}(A)=\frac{\partial^{n}}{\partial t_{1}, \ldots, \partial t_{n}} \operatorname{Prod}_{A}(0, \ldots, 0)
$$

Let us recall two famous results and one recent result by the author. 


\section{Van der Waerden Conjecture}

The famous Van der Waerden Conjecture [23] states that

$$
\min _{A \in \Omega_{n}} \operatorname{per}(A)=\frac{n !}{n^{n}}=: v d w(n) \quad(\text { VDW-bound })
$$

and the minimum is attained uniquely at the matrix $J_{n}$ in which every entry equals $\frac{1}{n}$. The Van der Waerden Conjecture was posed in 1926 and proved in 1981: D.I. Falikman proved in [5] the lower bound $\frac{n !}{n^{n}}$; the full conjecture, i.e. the uniqueness part, was proved by G.P. Egorychev in [4].

\section{Schrijver-Valiant Conjecture}

Define

$$
\begin{aligned}
\lambda(k, n) & =\min \left\{\operatorname{per}(A): A \in \Omega_{k, n}\right\}=k^{-n} \min \{\operatorname{per}(A): A \in \Lambda(k, n)\} ; \\
\theta(k) & =\lim _{n \rightarrow \infty}(\lambda(k, n))^{\frac{1}{n}} .
\end{aligned}
$$

It was proved in [26] that, using our notations, $\theta(k) \leq G(k)=:\left(\frac{k-1}{k}\right)^{k-1}$ and conjectured that $\theta(k)=G(k)$. Though the case of $k=3$ was proved by M. Voorhoeve in 1979 [28], this conjecture was settled only in 1998 [27] (17 years after the published proof of the Van der Waerden Conjecture). The main result of [27] is the remarkable (Schrijver-bound):

$$
\min \left\{\operatorname{per}(A): A \in \Omega_{k, n}\right\} \geq\left(\frac{k-1}{k}\right)^{(k-1) n}
$$

The proof of (Schrijver-bound) in [27] is, in the words of its author, "highly complicated".

Remark 1.1: The dynamics of research which led to (Schrijver-bound) is quite fascinating. If $k=2$ then $\min _{A \in \Lambda(2, n)} \operatorname{per}(A)=2$. Erdos and Renyi conjectured in 1968 paper that 3-regular case already has exponential growth:

$$
\min _{A \in \Lambda(3, n)} \operatorname{per}(A) \geq a^{n}, a>1 \text {. }
$$

This conjecture is implied by (VDW-bound), this connection was another important motivation for the Van der Waerden Conjecture. The Erdos-Renyi conjecture was answered by M. Voorhoeve in 1979 [28]:

$$
\min _{A \in \Lambda(3, n)} \operatorname{per}(A) \geq 6\left(\frac{4}{3}\right)^{n-3}
$$

Amazingly, the Voorhoeve's bound (5) is asymptotically sharp and the proof of this fact is probabilistic. In 1981 paper [26], A.Schrijver and W.G.Valiant found a sequence $\mu_{k, n}$ of probabilistic distributions on $\Lambda(k, n)$ such that

$$
\lim _{n \rightarrow \infty}\left(\min _{A \in \Lambda(k, n)} \operatorname{per}(A)\right)^{\frac{1}{n}} \leq \lim _{n \rightarrow \infty}\left(E_{\mu_{k, n}} \operatorname{per}(A)\right)^{\frac{1}{n}}=k\left(\frac{k-1}{k}\right)^{k-1}
$$


(I.M. Wanless recently extended in [30] the upper bound (6) to the boolean matrices in $\Lambda(k, n)$.)

It follows from the Voorhoeve's bound (5) that

$$
\lim _{n \rightarrow \infty}\left(E_{\mu_{k, n}} \operatorname{per}(A)\right)^{\frac{1}{n}}=\lim _{n \rightarrow \infty}\left(\min _{A \in \Lambda(k, n)} \operatorname{per}(A)\right)^{\frac{1}{n}} \quad \text { for } \quad k=2,3 .
$$

This was the rather bald intuition that gave rise to the Schrijver-Valiant 1981 conjecture.

The number $k\left(\frac{k-1}{k}\right)^{k-1}$ in Schrijver-Valiant conjecture came up via combinatorics followed by the standard Stirling's formula manipulations. On the other hand $G(k)=\left(\frac{k-1}{k}\right)^{k-1}=\frac{v d w(k)}{v d w(k-1)}$.

\section{Bapat's Conjecture (Van der Waerden Conjecture for mixed discrimi- nants)}

One of the problems posed in [2] is to determine the minimum of mixed discriminants of doubly stochastic tuples: $\min _{A \in D_{n}} D(A)=$ ?

Quite naturally, R.V.Bapat conjectured that $\min _{A \in D_{n}} D(A)=\frac{n !}{n^{n}}$ (Bapat-bound) and that it is attained uniquely at $\mathbf{J}_{n}=:\left(\frac{1}{n} I, \ldots, \frac{1}{n} I\right)$.

In [2] this conjecture was formulated for real matrices. The author had proved it [13] for the complex case, i.e. when matrices $A_{i}$ above are complex positive semidefinite and, thus, hermitian.

\subsection{The Ultimate Unification (and Simplification)}

Falikman/Egorychev proofs of the Van Der Waerden conjecture as well our proof of Bapat's conjecture are based on the Alexandrov inequalities for mixed discriminants [1] and some optimization theory, which is rather advanced in the case of the Bapat's conjecture. They all rely heavily on the matrix structure and essentially of non-inductive nature.

(D. I. Falikman independently rediscovered in [5] the diagonal case of the Alexandrov inequalities and used a clever penalty functional. The very short paper [5] is supremely original, it cites only three references and uses none of them.)

The Schrijver's proof has nothing in common with these analytic proofs; it is based on the finely tuned combinatorial arguments and multi-level induction. It heavily relies on the fact that the entries of matrices $A \in \Lambda(k, n)$ are integers.

The main result of this paper is one, easily stated and proved by easy induction, theorem which unifies, generalizes and, in the case of (Schrijver-bound), improves the results described above. This theorem is formulated in terms of the mixed derivative $\frac{\partial^{n}}{\partial x_{1} \ldots \partial x_{n}} p(0, \ldots, 0)$ (rewind to the formula $\left.(3)\right)$ of $\mathbf{H}-\mathbf{S t a b l e}$ (or positive hyperbolic) homogeneous polynomials $p$.

The next two completely self-contained sections introduce the basics of stable homogeneous polynomials and proofs of the theorem and its corollaries. We have tried to simplify 
everything to the undergraduate level, making the paper longer than a dry technical note of 4-5 pages. Our proof of the uniqueness in the generalized Van der Waerden Conjecture is a bit more involved, as it uses Garding's result on the convexity of the hyperbolic cone.

\section{Homogeneous Polynomials}

The next definition introduces key notations and notions.

\section{Definition 2.1:}

1. The linear space of homogeneous polynomials with real (complex) coefficients of degree $n$ and in $m$ variables is denoted $\operatorname{Hom}_{R}(m, n)\left(H_{C}(m, n)\right)$.

We denote as $\mathrm{Hom}_{+}(m, n)\left(\mathrm{Hom}_{++}(n, m)\right)$ the closed convex cone of polynomials $p \in \operatorname{Hom}_{R}(m, n)$ with nonnegative (positive) coefficients.

2. For a polynomial $p \in \mathrm{Hom}_{+}(n, n)$ we define its Capacity as

$$
\operatorname{Cap}(p)=\inf _{x_{i}>0, \prod_{1 \leq i \leq n} x_{i}=1} p\left(x_{1}, \ldots, x_{n}\right)=\inf _{x_{i}>0} \frac{p\left(x_{1}, \ldots, x_{n}\right)}{\prod_{1 \leq i \leq n} x_{i}} .
$$

3. Consider a polynomial $p \in \operatorname{Hom}_{C}(m, n)$,

$$
p\left(x_{1}, \ldots, x_{m}\right)=\sum_{\left(r_{1}, \ldots, r_{m}\right)} a_{r_{1}, \ldots, r_{m}} \prod_{1 \leq i \leq m} x_{i}^{r_{i}} .
$$

We define $\operatorname{Rank}_{p}(S)$ as the maximal joint degree attained on the subset $S \subset\{1, \ldots, m\}$ :

$$
\operatorname{Rank}_{p}(S)=\max _{a_{r_{1}, \ldots, r_{m}} \neq 0} \sum_{j \in S} r_{j}
$$

If $S=\{i\}$ is a singleton, we define $\operatorname{deg}_{p}(i)=\operatorname{Rank}_{p}(S)$.

4. Let $p \in \mathrm{Hom}_{+}(n, n)$,

$$
p\left(x_{1}, \ldots, x_{n}\right)=\sum_{r_{1}+\cdots+r_{n}=1} a_{r_{1}, \ldots, r_{n}} \prod_{1 \leq i \leq n} x_{i}^{r_{i}} .
$$

Such a homogeneous polynomial $p$ with nonnegative coefficients is called doublystochastic if

$$
\frac{\partial}{\partial x_{i}} p(1,1, \ldots, 1)=1: 1 \leq i \leq n
$$

In other words, $p \in \operatorname{Hom}_{+}(n, n)$ is doubly-stochastic if

$$
\sum_{r_{1}+\cdots+r_{n}=1} a_{r_{1}, \ldots, r_{n}} r_{j}=1: 1 \leq j \leq n
$$


It follows from the Euler's identity that $p(1,1, \ldots, 1)=1$ :

$$
\sum_{r_{1}+\cdots+r_{n}=1} a_{r_{1}, \ldots, r_{n}}=1
$$

Using the concavity of the logarithm on $R_{++}$we get that

$$
\log \left(p\left(x_{1}, \ldots, x_{n}\right)\right) \geq \sum_{r_{1}+\cdots+r_{n}=1} a_{r_{1}, \ldots, r_{n}} r_{i} \log \left(x_{i}\right)=\log \left(x_{1} \cdots x_{n}\right)
$$

Therefore

Fact 2.2: If $p \in \mathrm{Hom}_{+}(n, n)$ is doubly-stochastic then $\operatorname{Cap}(p)=1$.

5. A polynomial $p \in \operatorname{Hom}_{C}(m, n)$ is called $\mathbf{H}$-Stable if $p(Z) \neq 0$ provided $\operatorname{Re}(Z)>0$; is called $\mathbf{H}$-SStable if $p(Z) \neq 0$ provided $\operatorname{Re}(Z) \geq 0$ and $\sum_{1 \leq i \leq m} \operatorname{Re}\left(z_{i}\right)>0$.

We coined the term 'H-Stable" to stress two things: Homogeniety and Hurwitz' stability. Other terms are used in the same context: Wide Sense Stable in [15], Half-Plane Property in [3].

6. We define

$$
v d w(i)=\frac{i !}{i^{i}} ; G(i)=\frac{v d w(i)}{v d w(i-1)}=\left(\frac{i-1}{i}\right)^{i-1}, i>1 ; G(1)=1 .
$$

Notice that $v d w(i)$ as well as $G(i)$ are strictly decreasing sequences.

\section{Example 2.3:}

1. Let $p \in \mathrm{Hom}_{+}(2,2), p\left(x_{1}, x_{2}\right)=\frac{A}{2} x_{1}^{2}+C x_{1} x_{2}+\frac{B}{2} x_{2}^{2} ; A, B, C \geq 0$. Then $\operatorname{Cap}(p)=C+\sqrt{A B}$ and the polynomial $p$ is H-Stable iff $C \geq \sqrt{A B}$.

2. Let $A \in \Omega_{n}$ be a doubly stochastic matrix. Then the polynomial $\operatorname{Prod}_{A}$ is doublystochastic. Therefore $\operatorname{Cap}\left(\operatorname{Prod}_{A}\right)=1$. In the same way, if $\mathbf{A} \in D_{n}$ is a doubly stochastic $n$-tuple then the polynomial $\operatorname{Det}_{\mathbf{A}}$ is doubly-stochastic and $\operatorname{Cap}\left(\operatorname{Det}_{\mathbf{A}}\right)=$ 1.

3. Let $\mathbf{A}=\left(A_{1}, A_{2}, \ldots A_{m}\right)$ be an $m$-tuple of PSD hermitian $n \times n$ matrices, and $\sum_{1<i<m} A_{i} \succ 0$ (the sum is positive-definite). Then the determinantal polynomial $\operatorname{Det}_{\mathbf{A}}\left(t_{1}, \ldots, t_{m}\right)=\operatorname{det}\left(\sum_{1 \leq i \leq m} t_{i} A_{i}\right)$ is $\mathbf{H}$-Stable and

$$
\operatorname{Rank}_{\operatorname{Det}_{\mathbf{A}}}(S)=\operatorname{Rank}\left(\sum_{i \in S} A_{i}\right)
$$


The main result in this paper is the following Theorem.

Theorem 2.4: Let $p \in \mathrm{Hom}_{+}(n, n)$ be H-Stable polynomial. Then the following inequality holds

$$
\frac{\partial^{n}}{\partial x_{1} \ldots \partial x_{n}} p(0, \ldots, 0) \geq \prod_{2 \leq i \leq n} G\left(\min \left(i, \operatorname{deg}_{p}(i)\right)\right) \operatorname{Cap}(p)
$$

Note that

$$
\prod_{2 \leq i \leq n} G\left(\min \left(i, \operatorname{deg}_{p}(i)\right)\right) \geq \prod_{2 \leq i \leq n} G(i)=v d w(n)
$$

which gives the next generalized Van Der Waerden Inequality:

Corollary 2.5: Let $p \in \mathrm{Hom}_{+}(n, n)$ be $\mathbf{H}-$ Stable polynomial. Then

$$
\frac{\partial^{n}}{\partial x_{1} \ldots \partial x_{n}} p(0, \ldots, 0) \geq \frac{n !}{n^{n}} \operatorname{Cap}(p) .
$$

Corollary (2.5) was conjectured by the author in [10], where it was proved that $\frac{\partial^{n}}{\partial x_{1} \ldots \partial x_{n}} p(0, \ldots, 0) \geq C(n) C a p(p)$ for some constant $C(n)$.

\subsection{Three Conjectures/Inequalities}

The fundamental nature of Theorem (2.4) is illustrated in the following Example.

\section{Example 2.6:}

1. Let $A \in \Omega_{n}$ be $n \times n$ doubly stochastic matrix. It is easy to show that the polynomial $\operatorname{Prod}_{A}$ is H-Stable and doubly-stochastic. Therefore $\operatorname{Cap}\left(\operatorname{Prod}_{A}\right)=1$. Applying Corollary (2.5) we get the celebrated Falikman's result [5]:

$$
\min _{A \in \Omega_{n}} \operatorname{per}(A)=\frac{n !}{n^{n}}
$$

(The complementary uniqueness statement for Corollary (2.5) will be considered in $\operatorname{Section}(5)$.)

2. Let $\left(A_{1}, \ldots, A_{n}\right)=\mathbf{A} \in D_{n}$ be a doubly stochastic $n$-tuple. Then the determinantal polynomial $\operatorname{Det}_{\mathbf{A}}$ is $\mathbf{H}-\mathbf{S t a b l e}$ and doubly-stochastic. Thus $\operatorname{Cap}\left(\operatorname{Det}_{\mathbf{A}}\right)=1$ and we get the (Bapat-bound), proved by the author:

$$
\min _{\mathbf{A} \in D_{n}} D(\mathbf{A})=\frac{n !}{n^{n}}
$$


3. Important for what follows is the next observation, which is a diagonal case of (12): $\operatorname{deg}_{\operatorname{Prod}_{A}}(j)$ is equal to the number of nonzero entries in the $j$ th column of the matrix $A$.

The next Corrolary combines this observation with Theorem(2.4).

\section{Corollary 2.7:}

(a) Let $C_{j}$ be the number of nonzero entries in the $j$ th column of $A$, where $A$ is an $n \times n$ matrix with non-negative real entries. Then

$$
\operatorname{per}(A) \geq \prod_{2 \leq j \leq n} G\left(\min \left(j, C_{j}\right)\right) C a p\left(\operatorname{Prod}_{A}\right)
$$

(b) Suppose that $C_{j} \leq k: k+1 \leq j \leq n$. Then

$$
\operatorname{per}(A) \geq\left(\left(\frac{k-1}{k}\right)^{k-1}\right)^{n-k} \frac{k !}{k^{k}} \operatorname{Cap}\left(\operatorname{Prod}_{A}\right)
$$

Let $\Lambda(k, n)$ denote the set of $n \times n$ matrices with nonnegative integer entries and row and column sums all equal to $k$. The matrices in $\Lambda(k, n)$ correspond to the $k$-regular bipartite graphs with multiple edges.

Recall the (Schrijver-bound):

$$
\min _{A \in \Lambda(k, n)} \operatorname{per}(A) \geq k^{n} G(k)^{n}=\left(\frac{(k-1)^{k-1}}{k^{k-2}}\right)^{n} .
$$

The Falikman's inequality gives that

$$
\min _{A \in \Lambda(k, n)} \operatorname{per}(A) \geq k^{n} v d w(n)>k^{n} G(k)^{n} \quad \text { if } \quad k \geq n .
$$

Therefore the inequality (17) is interesting only if $k<n$.

Note that if $A \in \Lambda(k, n), k<n$ then all columns of $A$ have at most $k$ nonzero entries. If $A \in \Lambda(k, n)$ then the matrix $\frac{1}{k} A \in \Omega_{n}$, thus $\operatorname{Cap}\left(\operatorname{Prod}_{A}\right)=k^{n}$. As we observed above, $\operatorname{deg}_{\operatorname{Prod}_{A}}(j) \leq k$. Applying the inequality (16) to the polynomial $\operatorname{Prod}_{A}$ we get for $k<n$ an improved (Schrijver-bound):

$$
\min _{A \in \Lambda(k, n)} \operatorname{per}(A) \geq k^{n}\left(\left(\frac{k-1}{k}\right)^{k-1}\right)^{n-k} \frac{k !}{k^{k}}>\left(\frac{(k-1)^{k-1}}{k^{k-2}}\right)^{n} \text {. }
$$

Interestingly, the inequality (18) recovers for $k=3$ the Voorhoeve's inequality (5).

4. The inequality (15) is sharp if $C_{i}=\cdots=C_{n-1}=n ; C_{n}=k: 1<k \leq n-1$. To see this, consider the doubly stochastic matrix 


$$
D=\left(\begin{array}{cccc}
a & \ldots & a & b \\
\cdot & \ldots & . & . \\
a & \ldots & a & b \\
c & \ldots & c & 0 \\
\cdot & \ldots & . & . \\
c & \ldots & c & 0
\end{array}\right) ; a=\frac{1-b}{n-1}=\frac{k-1}{k(n-1)}, b=\frac{1}{k}, c=\frac{1}{n-1}
$$

and the associated polynomial

$$
\operatorname{Prod}_{D}\left(x_{1}, \ldots, x_{n}\right)=\left(\left(\sum_{1 \leq i \leq n-1} a x_{i}\right)+b x_{n}\right)^{k}\left(\sum_{1 \leq i \leq n-1} c x_{i}\right)^{n-k} .
$$

Since the matrix $D$ is doubly stochastic, $\operatorname{Cap}\left(\operatorname{Prod}_{D}\right)=1$. Direct inspection shows that

$$
\operatorname{per}(D)=(n-1) !(k b) a^{k-1} c^{n-k}=G(k) \frac{(n-1) !}{(n-1)^{n-1}} .
$$

Which gives the equality

$$
\operatorname{per}(D)=C a p\left(\operatorname{Prod}_{D}\right) \prod_{2 \leq j \leq n} G\left(\min \left(j, C_{j}\right)\right) .
$$

It follows that $\min \left\{\operatorname{per}(A): A \in \Omega_{n}^{(0)}\right\}=\frac{(n-1) !}{(n-1)^{n-1}}\left(\frac{n-2}{n-1}\right)^{n-2}$, where $\Omega_{n}^{(0)}$ is the set of $n \times n$ doubly stochastic matrices with at least one zero entry.

\section{$2.2 \quad$ The Main Idea}

Let $p \in \mathrm{Hom}_{+}(n, n)$. Define the following polynomials $q_{i} \in \mathrm{Hom}_{+}(i, i)$ :

$$
q_{n}=p, q_{i}\left(x_{1}, \ldots, x_{i}\right)=\frac{\partial^{n-i}}{\partial x_{i+1} \ldots \partial x_{n}} p\left(x_{1}, \ldots, x_{i}, 0, \ldots, 0\right) ; 1 \leq i \leq n-1 .
$$

Notice that $q_{1}\left(x_{1}\right)=\frac{\partial^{n}}{\partial x_{1} \ldots \partial x_{n}} p(0) x_{1}$ and

$$
q_{2}\left(x_{1}, x_{2}\right)=\frac{\partial^{n}}{\partial x_{1} \ldots \partial x_{n}} p(0) x_{1} x_{2}+\frac{1}{2}\left(\frac{\partial^{n}}{\partial x_{1} \partial x_{1} \ldots \partial x_{n}} p(0) x_{1}^{2}+\frac{\partial^{n}}{\partial x_{2} \partial x_{2} \ldots \partial x_{n}} p(0) x_{2}^{2}\right) \text {. }
$$

Therefore, $\operatorname{Cap}\left(q_{1}\right)=\frac{\partial^{n}}{\partial x_{1} \ldots \partial x_{n}} p(0)$ and

$$
\operatorname{Cap}\left(q_{2}\right)=\frac{\partial^{n}}{\partial x_{1} \ldots \partial x_{n}} p(0)+\sqrt{\frac{\partial^{n}}{\partial x_{1} \partial x_{1} \ldots \partial x_{n}} p(0) \frac{\partial^{n}}{\partial x_{2} \partial x_{2} \ldots \partial x_{n}} p(0) .}
$$


is

Define the univariate polynomial $R(t)=p\left(x_{1}, \ldots, x_{n-1}, t\right)$. Then its derivative at zero

$$
R^{\prime}(0)=q_{n-1}\left(x_{1}, \ldots, x_{n-1}\right) .
$$

Another simple but important observation is the next inequality:

$$
\operatorname{deg}_{q_{i}}(i) \leq \min \left(i, \operatorname{deg}_{p}(i)\right) \Longleftrightarrow G\left(\operatorname{deg}_{q_{i}}(i)\right) \geq G\left(\min \left(i, \operatorname{deg}_{p}(i)\right)\right): 1 \leq i \leq n .
$$

Recall that $v d w(i)=\frac{i !}{i^{i}}$. Suppose that the next inequalities hold

$$
\operatorname{Cap}\left(q_{i-1}\right) \geq \operatorname{Cap}\left(q_{i}\right) \frac{v d w(i)}{v d w(i-1)}=\operatorname{Cap}\left(q_{i}\right) G(i): 2 \leq i \leq n .
$$

Or better, the next stronger ones hold

$$
\operatorname{Cap}\left(q_{i-1}\right) \geq \operatorname{Cap}\left(q_{i}\right) G\left(\operatorname{deg}_{q_{i}}(i)\right): 2 \leq i \leq n,
$$

where

$$
G(m)=\frac{v d w(m)}{v d w(m-1)}=\left(\frac{m-1}{m}\right)^{m-1} .
$$

The next result, proved by the straigthforward induction, summarizes the main idea of our approach.

\section{Theorem 2.8:}

1. If the inequalities (24) hold then the next generalized Van Der Waerden inequality holds:

$$
\frac{\partial^{n}}{\partial x_{1} \ldots \partial x_{n}} p(0, \ldots, 0)=\operatorname{Cap}\left(q_{1}\right) \geq v d w(n) \operatorname{Cap}(p) .
$$

In the same way, the next inequality holds for Cap $\left(q_{2}\right)$ :

$$
\frac{\partial^{n}}{\partial x_{1} \ldots \partial x_{n}} p(0)+\sqrt{\frac{\partial^{n}}{\partial x_{1} \partial x_{1} \ldots \partial x_{n}} p(0) \frac{\partial^{n}}{\partial x_{2} \partial x_{2} \ldots \partial x_{n}} p(0)} \geq 2 v d w(n) \operatorname{Cap}(p) .
$$

2. If the inequalities (25) hold then the next generalized (Schrijver-bound) holds:

$$
\frac{\partial^{n}}{\partial x_{1} \ldots \partial x_{n}} p(0, \ldots, 0)=\operatorname{Cap}\left(q_{1}\right) \geq \operatorname{Cap}(p) \prod_{2 \leq i \leq n} G\left(\min \left(i, \operatorname{deg}_{p}(i)\right)\right) .
$$

What is left is to prove that the inequalities (25) hold for $\mathbf{H}$-Stable polynomials. We break the proof of this statement in two steps.

1. Prove that if $p \in \mathrm{Hom}_{+}(n, n)$ is $\mathbf{H}$-Stable then $q_{n-1}$ is either zero or $\mathbf{H}$-Stable. Using equation (22), this implication follows from Gauss-Lukas Theorem. GaussLukas Theorem states that if $z_{1}, \ldots, z_{n} \in C$ are the roots of an univariate polynomial $Q$ then the roots of its derivative $Q^{\prime}$ belong to the convex hull $C O\left(\left\{z_{1}, \ldots, z_{n}\right\}\right)$.

This step is, up to minor perturbation arguments, known. See, for instance, [16]. The result in [16] is stated in terms of hyperbolic polynomials, see Remark (5.2) for the connection between $\mathbf{H}-\mathbf{S t a b l e}$ and hyperbolic polynomials. Our treatment, described in Section(4), is self-contained, short and elementary. 
2. Prove that $\operatorname{Cap}\left(q_{n-1}\right) \geq G\left(\operatorname{deg}_{p}(n)\right) \operatorname{Cap}(p)$. This inequality boils down to the next inequality for the univariate polynomial $R$ from (22):

$$
R^{\prime}(0) \geq G(\operatorname{deg}(R))\left(\inf _{t>0} \frac{R(t)}{t}\right) .
$$

We prove it using AM/GM inequality and the fact that the roots of the polynomial $R$ are real.

It is instructive to see what is going on in the "permanental case": we start with the polynomial $\operatorname{Prod}_{A}$ which is a product of nonnegative linear forms. The very first polynomial in the induction, $q_{n-1}$, is not of this type in the generic case. I.e. there is no one matrix/graph associated with $q_{n-1}$. We gave up the matrix structure but had won the game.

In the rest of the paper Facts are statements which are quite simple and (most likely) known. We included them having in mind the undergraduate student reader.

\section{Univariate Polynomials}

\section{Proposition 3.1:}

1. (Gauss-Lukas Theorem)

Let $R(z)=\sum_{0 \leq i \leq n} a_{i} z^{i}$ be a Hurwitz polynomial with complex coefficients, i.e. all the roots of $R$ have negative real parts.

Then its derivative $R^{\prime}$ is Hurwitz.

2. Let $R(z)=\sum_{0 \leq i \leq n} a_{i} z^{i}$ be a Hurwitz polynomial with real coefficients and $a_{n}>0$. Then all the coefficients are positive real numbers.

\section{Proof:}

1. Recall that

$$
\frac{R^{\prime}(z)}{R(z)}=\sum_{1 \leq j \leq n} \frac{1}{z-z_{j}}
$$

Let $\mu$ be a root of $R^{\prime}$. Consider two cases. First: $\mu$ is a root of $R$. Then clearly $R e(\mu)<0$. Second: $\mu$ is not a root of $R$. Then

$$
L=: \sum_{1 \leq j \leq n} \frac{1}{\mu-z_{j}}=0 .
$$

Suppose that $\operatorname{Re}(\mu) \geq 0$. As $(a+i b)^{-1}=\frac{a-i b}{a^{2}+b^{2}}$ we get that

$$
\operatorname{Re}\left(\frac{1}{\mu-z_{j}}\right)=\frac{\operatorname{Re}(\mu)-\operatorname{Re}\left(z_{j}\right)}{\left(\operatorname{Re}(\mu)-\operatorname{Re}\left(z_{j}\right)\right)^{2}+\left(\operatorname{Im}(\mu)-\operatorname{Im}\left(z_{j}\right)\right)^{2}}>0 .
$$

Therefore $\operatorname{Re}(L)>0$ which leads to a contradiction. Thus $\operatorname{Re}(\mu)<0$ and the derivative $R^{\prime}$ is Hurwitz. 
2. This part is easy and well known.

The next simple result binds together all the small pieces of our approach.

Lemma 3.2: Let $Q(t)=\sum_{0 \leq i \leq k} a_{i} t^{i} ; a_{k}>0, k \geq 2$ be a polynomial with non-negative coefficients and real (non-positive) roots. Define $C=\inf _{t>0} \frac{Q(t)}{t}$. Then the next inequlity holds:

$$
a_{1}=Q^{\prime}(0) \geq\left(\frac{k-1}{k}\right)^{k-1} C
$$

The equality holds if and only if all the roots of $Q$ are equal negative numbers, i.e. $Q(t)=$ $b(t+a)^{k}$ for some $a, b>0$.

Proof: If $Q(0)=0$ then $Q^{\prime}(0) \geq C>\left(\frac{k-1}{k}\right)^{k-1} C$.

Let $Q(0)>0$. We then can assume WLOG that $Q(0)=1$. In this case all the roots of $Q$ are negative real numbers. Thus

$$
Q(t):=\prod_{i=1}^{k}\left(a_{i} t+1\right): a_{i}>0,1 \leq i \leq k
$$

and $Q^{\prime}(0)=a_{1}+\cdots+a_{k}$.

Using the AM/GM inequality we get that

$$
C t \leq Q(t) \leq P(t)=:\left(1+\frac{Q^{\prime}(0)}{k} t\right)^{k}, t \geq 0 .
$$

It follows from basic calculus that

$$
\inf _{t>0} \frac{P(t)}{t}=P(s)=Q^{\prime}(0)\left(\frac{k}{k-1}\right)^{k-1}, \quad \text { where } \quad s=\frac{k}{Q^{\prime}(0)(k-1)} .
$$

Therefore

$$
C \leq \inf _{t>0} \frac{P(t)}{t}=Q^{\prime}(0)\left(\frac{k}{k-1}\right)^{k-1},
$$

which finally yields the desired inequality

$$
Q^{\prime}(0) \geq\left(\frac{k-1}{k}\right)^{k-1} C, k \geq 2 .
$$

It follows from the uniqueness condition in the AM/GM inequality that the equality in (30) holds if and only if $0<a_{1}=\cdots=a_{k}$. 
Remark 3.3: The condition that the roots of $Q$ are real can be relaxed in several ways. For instance the statement of Lemma (3.2) holds for any map $f: R_{+} \rightarrow R_{+}$such that the derivative $f^{\prime}(0)$ exists and $f^{\frac{1}{k}}$ is concave.

If such map is $\log$-concave, i.e $\log (f)$ is concave, then $f^{\prime}(0) \geq \frac{1}{e} \inf _{t>0} \frac{f(t)}{t}$.

Notice that the right inequality in (31) is essentially equivalent to the concavity of the function $(Q(t))^{\frac{1}{k}}$ on $R_{+}$.

It was shown in [11] that the inequality (30) is equivalent to the (VDW-bound) for doubly stochastic matrices $A \in \Omega_{n}: A=[a|b| \ldots \mid b]$ with two distinct columns.

\section{Stable homogeneous polynomials}

\subsection{Basics}

Definition 4.1: A polynomial $p \in \operatorname{Hom}_{C}(m, n)$ is called H-Stable if $p(Z) \neq 0$ provided $\operatorname{Re}(Z)>0$; is called $\mathbf{H}-\mathbf{S S t a b l e}$ if $p(Z) \neq 0$ provided $\operatorname{Re}(Z) \geq 0$ and $\sum_{1 \leq i \leq m} \operatorname{Re}\left(z_{i}\right)>0$.

Fact 4.2: Let $p \in \operatorname{Hom}_{C}(m, n)$ be $\mathbf{H}$-Stable and $A$ is $m \times m$ matrix with nonnegative real entries without zero rows. Then the polynomial $p_{A}$, defined as $p_{A}(Z)=p(A Z)$ is also $\mathbf{H}-\mathbf{S t a b l e . ~ I f ~ a l l ~ e n t r i e s ~ o f ~} A$ are positive then $p_{A}$ is $\mathbf{H}$-SStable.

Fact 4.3: Let $p \in \operatorname{Hom}_{C}(m, n), Y \in C^{m}, p(Y) \neq 0$. Define the following univariate polynomial of degree $n$ :

$$
L_{X, Y}(t)=p(t Y-X)=p(Y) \prod_{1 \leq i \leq n}\left(t-\lambda_{i ; Y}(X)\right): X \in C^{m} .
$$

Then

$$
\lambda_{i ; Y}(b X+a Y)=b \lambda_{i ; Y}(X)+a ; p(X)=p(Y) \prod_{1 \leq i \leq n} \lambda_{i ; Y}(X) .
$$

The following simple result substantially simplifies the proofs below. Proposition (4.4) connects the notion of $\mathbf{H}$-Stability with the notion of Hyperbolicity, see more on this connection in Subsection(5.1).

Proposition 4.4: A polynomial $p \in \mathrm{Hom}_{C}(m, n)$ is $\mathbf{H}$-Stable if and only if $p(X) \neq$ $0: X \in R_{++}^{m}$ and the roots of univariate polynomials $P(t X-Y): X, Y \in R_{++}^{m}$ are real positive numbers.

\section{Proof:}

1. Suppose that $p(X) \neq 0: X \in R_{++}^{m}$ and the roots of univariate polynomials $p(t X-$ $Y): X, Y \in R_{++}^{m}$ are real positive numbers. It follows from identities (32) (shift $L \rightarrow L+a X>0)$ that the roots of $P(t X-L): X \in R_{++}^{m}, L \in R^{m}$ are real numbers. We want to prove that this property implies that $p \in \operatorname{Hom}_{C}(m, n)$ is 
H-Stable. Let $Z=\operatorname{Re}(Z)+i \operatorname{Im}(Z) \in C^{m}: \operatorname{Im}(Z) \in R^{m}, 0<\operatorname{Re}(Z) \in R_{++}^{m}$. If $p(Z)=0$ then also $p(-i \operatorname{Re}(Z)+\operatorname{Im}(Z))=0$, which contradicts the real rootedness of $p(t X-Y): X>0, Y \in R^{m}$.

2. Suppose that $p \in \operatorname{Hom}_{C}(m, n)$ is H-Stable. Let $X, Y \in R_{++}^{m}$ and $p(z X-Y)=$ $0, z=a+b i$. We need to prove that $b=0$ and $a>0$. If $b \neq 0$ then $p(a X-Y+b i X)=$ $(b i)^{n} p\left(X-b^{-1} i(a X-y)\right) \neq 0$ as the real part $\operatorname{Re}\left(X-b^{-1} i(a X-y)\right)=X>0$. Therefore $b=0$. If $a \leq 0$ then $-(a X-Y) \in R_{++}^{m}$. Which implies that $p(a X-Y)=$ $(-1)^{n} p(-(a X-Y)) \neq 0$. Thus $a>0$.

We will use the following corollaries:

Corollary 4.5: If $\operatorname{Re}(Z) \in R_{+}^{m}$ and a polynomial $p$ is $\mathbf{H}$-Stable then

$$
|p(Z)| \geq|p(\operatorname{Re}(Z))| .
$$

Proof: Since $p$ is continuous on $C^{m}$ hence it is sufficient to assume that $\operatorname{Re}(Z) \in R_{++}^{m}$. It follows from identities (32) that

$$
p(Z)=p(\operatorname{Re}(Z)+i \operatorname{Im}(Z))=p(\operatorname{Re}(Z)) \prod_{1 \leq j \leq n}\left(1+i \lambda_{j}\right),
$$

where $\left(\lambda_{1}, \ldots, \lambda_{n}\right)$ are the roots of the univariate polynomial $p(\operatorname{Re}(Z)-\operatorname{Im}(Z))$. Because $\operatorname{Re}(Z) \in R_{++}^{m}$, all these roots are real numbers.

Therefore $|p(Z)|=|p(\operatorname{Re}(Z))| \prod_{1 \leq j \leq n}\left|1+i \lambda_{j}\right| \geq|p(\operatorname{Re}(Z))|$.

Corollary 4.6: Let $p \in \operatorname{Hom}_{C}(m, n)$ be H-Stable; $X, Y \in R^{m}$ and $0<X+Y \in R_{++}^{m}$. Then all the roots of the univariate polynomial equation $p(t X+Y)=0$ are real numbers.

Proof: Let $p(t X+Y)=0$, then also $p((t-1) X+(X+Y))=0$. Since $X+Y>0$ hence $t-1 \neq 0$. As the polynomial $p$ is homogeneous therefore $p\left(X+(1-t)^{-1}(X+Y)\right)=0$. It follows that $(1-t)^{-1}$ is real, thus $t$ is also a real number.

Fact 4.7: Let $p \in H_{o m}(m, n)$ be H-SStable (H-Stable). Then for all $X \in R_{++}^{m}$ the coefficients of the polynomial $q=\frac{p}{p(X)}$ are positive (nonnegative) real numbers.

Proof: We prove first the case of $\mathbf{H}$-SStable polynomials.

Since $q(X)=1$ we get from (32) that $q(Y)$ is a positive real number for all vectors $Y \in R_{++}^{m}$. Therefore, by a standard interpolation argument, the coefficients of $q$ are real. We will prove by induction the following equivalent statement: if $q \in \operatorname{Hom}_{R}(m, n)$ is $\mathbf{H}$ SStable and $q(Y)>0$ for all $Y \in R_{++}^{m}$ then the coefficients of $q$ are all positive. Write $q(t ; Z)=\sum_{0 \leq i \leq n} t^{i} q_{i}(Z)$, where $Z \in C^{m-1}$, the polynomials $q_{i} \in H_{0} m_{R}(m-1, n-i)$, $0 \leq i \leq n-1$ and $q_{n}(Z)$ is a real number. Let us fix the complex vector $Z$ such that $\operatorname{Re}(Z) \in R_{+}^{m-1}$ and $\operatorname{Re}(Z) \neq 0$. Since $q$ is $\mathbf{H}$-SStable hence all roots of the univariate polynomial $q(t ; Z)$ have negative real parts. Therefore, using the first part of Proposition 
(3.1), we get that polynomials $q_{i}: 0 \leq i \leq n$ are all H-SStable. Since the degree of $q$ is $n$ hence $q_{n}(Z)$ is a constant, $q_{n}(Z)=q(1 ; 0)>0$. Using now the second part of Proposition (3.1), we see that $q_{i}(Y)>0$ for all $Y \in R_{++}^{m}$ and $0 \leq i \leq n$. Continuing this process we will end up with either $m=1$ or $n=1$. Both those cases have positive coefficients.

Let $p \in \operatorname{Hom}_{C}(m, n)$ be $\mathbf{H}$-Stable and $A>0$ is $m \times m$ matrix with positive entries such that $A X=X$. Then for all $\epsilon>0$ the polynomials $q_{I+\epsilon A} \in \operatorname{Hom}_{R}(m, n)$, defined as in Fact(4.2), are H-SStable and $\lim _{\epsilon \rightarrow 0} q_{I+\epsilon A}=q$. Therefore the coefficients of $q$ are nonnegative real numbers.

From now on we will deal only with the polynomials with nonnegative coefficients.

Corollary 4.8: Let $p_{i} \in \mathrm{Hom}_{+}(m, n)$ be a sequence of $\mathbf{H}-\mathbf{S t a b l e ~ p o l y n o m i a l s ~ a n d ~} p=$ $\lim _{i \rightarrow \infty} p_{i}$. Then $p$ is either zero or $\mathbf{H}-\mathbf{S t a b l e . ~}$

Some readers might recognize Corollary (4.8) as a particular case of A. Hurwitz's theorem on limits of sequences of nowhere zero analytical functions. Our proof below is elementary.

Proof: Suppose that $p$ is not zero. Since $p \in \operatorname{Hom}_{+}(m, n)$ hence $p\left(x_{1}, \ldots, x_{m}\right)>0$ if $x_{j}>0: 1 \leq j \leq m$. As the polynomials $p_{i}$ are $\mathbf{H}$-Stable therefore $\left|p_{i}(Z)\right| \geq\left|p_{i}(\operatorname{Re}(Z))\right|$ : $\operatorname{Re}(Z) \in R_{++}^{m}$. Taking the limits we get that $|p(Z)| \geq|p(\operatorname{Re}(Z))|>0: \operatorname{Re}(Z) \in R_{++}^{m}$, which means that $p$ is $\mathbf{H}$-Stable.

Fact 4.9: For a polynomial $p \in \operatorname{Hom}_{C}(m, n)$ we define a polynomial $q \in \mathrm{Hom}_{C}(m-1, n-1)$ as

$$
q\left(x_{1}, \ldots, x_{m-1}\right)=\frac{\partial}{\partial x_{m}} p\left(x_{1}, \ldots, x_{m-1}, 0\right) .
$$

Then the next two statements hold:

1. Let $p \in \mathrm{Hom}_{+}(m, n)$ be $\mathbf{H}$-SStable. Then the polynomial $q$ is also H-SStable.

2. Let $p \in \operatorname{Hom}_{+}(m, n)$ be $\mathbf{H}$-Stable. Then the polynomial $q$ is either zero or $\mathbf{H}$ Stable.

\section{Proof:}

1. Let $p \in \operatorname{Hom}_{+}(m, n)$ be $\mathbf{H}-\mathbf{S S t a b l e}$ and consider an univariate polynomial

$$
R(z)=p(Y ; z): z \in C, Y \in C^{m-1} .
$$

Suppose that $0 \neq \operatorname{Re}(Y) \geq 0$. It follows from the definition of H-SStability that $R(z) \neq 0$ if $\operatorname{Re}(z) \geq 0$. In other words, the univariate polynomial $R$ is Hurwitz. It follows from Gauss-Lukas Theorem that

$$
q(Y)=R^{\prime}(0) \neq 0
$$

which means that $q$ is $\mathbf{H}-\mathbf{S S t a b l e . ~}$ 
2. Let $p \in \operatorname{Hom}_{+}(m, n)$ be H-Stable and $q \neq 0$. Take an $m \times m$ matrix $A>0$. Then the polynomial $p_{I+\epsilon A}, p_{I+\epsilon A}(Z)=p((I+\epsilon A) Z)$ is $\mathbf{H}$-SStable for all $\epsilon>0$. Therefore, using the first part, $q_{I+\epsilon A}$ is H-SStable. Clearly $\lim _{\epsilon \rightarrow 0} q_{I+\epsilon A}=q$. Since $q \neq 0$, it follows from Corollary (4.8) that $q$ is H-Stable.

Theorem 4.10: Let $p \in \operatorname{Hom}_{+}(n, n)$ be $\mathbf{H}-$ Stable, and

$$
q_{n-1}\left(x_{1}, \ldots, x_{n-1}\right)=\frac{\partial}{\partial x_{n}} p\left(x_{1}, \ldots, x_{n-1}, 0\right) .
$$

Then

$$
\operatorname{Cap}\left(q_{n-1}\right) \geq \operatorname{Cap}(p) G\left(\operatorname{deg}_{p}(n)\right) .
$$

Proof: We need to prove that

$$
\frac{\partial}{\partial x_{n}} p\left(x_{1}, \ldots, x_{n-1}, 0\right) \geq \operatorname{Cap}(p) G\left(\operatorname{deg}_{p}(n)\right), x_{1}, \ldots, x_{n-1}>0, \prod_{1 \leq i \leq n-1} x_{i}=1 .
$$

Fix a positive vector $\left(x_{1}, \ldots, x_{n-1}\right), \prod_{1 \leq i \leq n-1} x_{i}=1$ and define, as in proof of Fact (4.9), the polynomial $R(t)=p\left(x_{1}, \ldots, x_{n-1}, t\right)$. It follows from Corollary(4.6) that all the roots of $R$ are real. Since the coefficients of the polynomial $R$ are non-negative hence its roots are non-positive real numbers. It follows from a definition of $\operatorname{Cap}(p)$ that $R(t) \geq C a p(p) t$, therefore

$$
\inf _{t>0} \frac{R(t)}{t} \geq \operatorname{Cap}(p) .
$$

The degree of the polynomial $R$ is equal to $\operatorname{deg}_{p}(n)$. It finally follows from Lemma(3.2) that

$$
q_{n-1}\left(x_{1}, \ldots, x_{n-1}, 0\right)=R^{\prime}(0) \geq \operatorname{Cap}(p) G\left(\operatorname{deg}_{p}(n)\right) .
$$

\section{Uniqueness in Generalized Van Der Waerden In- equality}

\subsection{Hyperbolic Polynomials}

The following concept of hyperbolic polynomials arose from the theory of partial differential equations [6], [14]. A recent paper [24] gives nice and concise introduction to the area (with simplified proofs of the key theorems) and describes connections to convex optimization. 


\section{Definition 5.1:}

1. A homogeneous polynomial $p: C^{m} \rightarrow C$ of degree $n\left(p \in \operatorname{Hom}_{C}(m, n)\right)$ is called hyperbolic in the direction $e \in R^{m}$ (or e-hyperbolic) if $p(e) \neq 0$ and for each vector $X \in R^{m}$ the univariate (in $\left.\lambda\right)$ polynomial $p(X-\lambda e)$ has exactly $n$ real roots counting their multiplicities.

2. Denote an ordered vector of roots of $p(x-\lambda e)$ as

$$
\lambda_{e}(X)=\left(\lambda_{n}(X) \geq \lambda_{n-1}(X) \geq \ldots \lambda_{1}(X)\right) .
$$

Call $X \in R^{m} e$-positive (e-nonnegative) if $\lambda_{1}(X)>0\left(\lambda_{n}(X) \geq 0\right)$. We denote the closed set of $e$-nonnegative vectors as $N_{e}(p)$, and the open set of $e$-positive vectors as $C_{e}(p)$.

Remark 5.2: Proposition (4.4) essentially says that a polynomial $p \in \operatorname{Hom}_{C}(m, n)$ is $\mathbf{H}$-Stable iff $p$ is hyperbolic in some direction $e \in R_{++}^{m}$ and the inclusion $R_{+}^{m} \subset N_{e}(p)$ holds. If $p \in \operatorname{Hom}_{C}(m, n)$ is $\mathbf{H}$-SStable then any non-zero vector $0 \leq X \in R_{+}^{m}$ belongs to the (open) hyperbolic cone $C_{e}(p)$.

We need the next fundamental fact due to L. Garding [6] (we recommend the very readable treatment in $[24])$ :

Theorem 5.3: Let $p \in \operatorname{Hom}_{C}(m, n)$ be e-hyperbolic polynomial and $d \in C_{e}(p) \subset R^{m}$. Then $p$ is also d-hyperbolic and $C_{d}(p)=C_{e}(p), N_{d}(p)=N_{e}(p)$. Moreover cone $C_{e}(p)$, called hyperbolic cone, is convex.

\section{Corollary 5.4:}

1. For any two vectors in the hyperbolic cone $d_{1}, d_{2} \in C_{e}(p)$ the following set equality holds:

$$
N_{d_{1}}(p) \bigcap\left(-N_{d_{1}}(p)\right)=N_{d_{2}}(p) \bigcap\left(-N_{d_{2}}(p)\right)=N u l l_{p}
$$

Thus Null $_{p} \subset R^{m}$ is a linear subspace.

2.

$$
\text { Null }_{p}=\left\{X \in R^{m}: p(Y+X)=p(Y) \text { for all } Y \in C^{m}\right\}
$$

Let $\operatorname{Pr}\left(N_{u l l}\right)$ be orthogonal projector on the linear subspace $N u l l_{p}$. It follows from (37) that

$$
p(Y)=p\left(\left(I-\operatorname{Pr}\left(N u l l_{p}\right)\right) Y\right)
$$

3. Let $p \in \operatorname{Hom}_{+}(n, n)$ be a doubly-stochastic $\mathbf{H}$-Stable polynomial. If $\left(y_{1}, \ldots, y_{n}\right) \in$ $\mathrm{Null}_{p}$ then

$$
y_{1}+\cdots+y_{n}=0
$$




\section{Proof:}

1. It is well known and obvious that if $K$ is a convex cone in some linear space $L$ over reals then the intersection $K \bigcap(-K)$ is a linear subspace of $L$.

2. Let $T \in C_{e}(p)$ and $X \in N u l_{p}$. Then all the roots of the equation $p(x T+X)=0$ are equal to zero. Since $p(T) \neq 0$ and the polynomial $p \in H_{C}(m, n)$ is homogeneous, hence $p(x T+X)=x^{n} p(T)$. Therefore $p(T+X)=p(T)$ for all $T \in C_{e}(p)$. As $C_{e}(p)$ is a non-empty open subset of $R^{m}$, equality (37) follows from the analyticity of $p$.

3. Consider the vector of all ones $e=(1, \ldots, 1) \in R^{n}$ and a vector $Y=\left(y_{1}, \ldots, y_{n}\right) \in$ $N u l l_{p}$. Then $d(t)=p(e+t Y)=p(e)$ for all $t \in R$. Therefore

$$
0=d^{\prime}(0)=\sum_{1 \leq i \leq n} y_{i} \frac{\partial}{\partial x_{i}} p(1,1, \ldots, 1)=y_{1}+\cdots+y_{n}
$$

\section{Example 5.5:}

1. Consider the power polynomial $q \in \operatorname{Hom}_{+}(n, n), q\left(x_{1}, \ldots, x_{n}\right)=\left(a_{1} x_{1}+\cdots+a_{n} x_{n}\right)^{n}$. If the non-zero vector $\mathbf{a}=\left(a_{1}, \ldots, a_{n}\right) \in R_{+}^{n}$ then the power polynomial $q$ is $\mathbf{H}$ Stable. The correspondind linear subspace

$$
\operatorname{Null}_{p}=\mathbf{a}^{\perp}=:\left\{\left(y_{1}, \ldots, y_{n}\right) \in R^{n}: \sum_{1 \leq i \leq n} a_{i} y_{i}=0\right\}, \quad \operatorname{dim}\left(N u l l_{p}\right)=n-1
$$

and $\operatorname{Cap}(p)=n^{n} \prod_{1 \leq i \leq n} a_{i}$. Therefore $\operatorname{Cap}(q) \neq 0$ iff $\mathbf{a} \in R_{++}^{n}$.

It is easy to see that

$$
\frac{\partial^{n}}{\partial x_{1} \ldots \partial x_{n}}\left(a_{1} x_{1}+\cdots+a_{n} x_{n}\right)^{n}=n ! a_{1} \ldots a_{n} .
$$

Therefore

$$
\frac{\partial^{n}}{\partial x_{1} \ldots \partial x_{n}} q(0, \ldots, 0)=\operatorname{Cap}(q) \frac{n !}{n^{n}} .
$$

If $\operatorname{dim}\left(\operatorname{Null}_{p}\right)=n-1$ and a polynomial $p \in \operatorname{Hom}_{+}(n, n)$ is H-Stable then $p\left(x_{1}, \ldots, x_{n}\right)=\left(b_{1} x_{1}+\cdots+b_{n} x_{n}\right)^{n}$ for some non-zero vector $\mathbf{b}=\left(b_{1}, \ldots, b_{n}\right) \in R_{+}^{n}$. The power polynomial $p$ is doubly-stochastic iff $b_{i}=\frac{1}{n}, 1 \leq i \leq n$.

2. Let $p \in \operatorname{Hom}_{C}(m, n)$ be an $e$-hyperbolic polynomial, $D \in C_{e}(p) \subset R^{m}$ and $X \in R^{m}$. Suppose that the univariate polynomial $R(t)=p(t D+X)=a(t+b)^{n}, b \in R$. Define the next real vector $Y=-b D+X$. Then all the roots of the equation $p(Y-\lambda D)=0$ are equal to zero. Therefore $Y \in N_{D}(p) \bigcap\left(-N_{D}(p)\right)=N u l l_{p}$. 


\subsection{Uniqueness}

Definition 5.6: We call a H-Stable polynomial $p \in H_{\circ}(n, n)$ extremal if $\operatorname{Cap}(p)>0$ and

$$
\frac{\partial^{n}}{\partial x_{1} \ldots \partial x_{n}} p(0, \ldots, 0)=\frac{n !}{n^{n}} \operatorname{Cap}(p)
$$

Our goal is the next theorem

Theorem 5.7: A H-Stable polynomial $p \in \operatorname{Hom}_{+}(n, n)$ is extremal if and only if

$$
p\left(x_{1}, \ldots, x_{n}\right)=\left(a_{1} x_{1}+\cdots+a_{n} x_{n}\right)^{n}
$$

for some positive real numbers $a_{1}, \ldots, a_{n}>0$.

In other words, the equality (41) holds iff $\operatorname{dim}\left(N u l l_{p}\right)=n-1$.

Notice that the "if" part is simple and follows from the equality(40).

We collect the basic properties of extremal polynomials in the next proposition.

\section{Proposition 5.8:}

1. If a $\mathbf{H}-$ Stable polynomial $p \in \operatorname{Hom}_{+}(n, n)$ is extremal then all its coefficients are positive real numbers.

2. Let $\mathbf{c}=\left(c_{1}, \ldots, c_{n}\right) \in R_{++}^{n}$ and $p \in \operatorname{Hom}_{+}(n, n)$. Define the scaled polynomial $p_{\mathbf{c}}$ as $p_{\mathbf{c}}\left(x_{1}, \ldots, x_{n}\right)=p\left(c_{1} x_{1}, \ldots, c_{n} x_{n}\right)$. If $p \in H_{o m_{+}}(n, n)$ is H-Stable extremal polynomial then also the scaled polynomial $p_{\mathbf{c}}$ is.

\section{Proof:}

1. Our goal in this step is to show that if equation (41) holds then the H-Stable polynomial $p$ is, in fact, H-SStable and therefore has all positive coefficients.

Since $G(2) \cdots G(n)=\frac{n !}{n^{n}}$ and the function $G$ is strictly decreasing on $[0, \infty)$, hence it follows from (13) that $\operatorname{deg}_{p}(n)=n$. Since the inequality (13) is invariant with respect to permutations of variables hence $\operatorname{deg}_{p}(i)=n: 1 \leq i \leq n$. Which means that $p\left(e_{i}\right)>0: 1 \leq i \leq n$, where $\left\{e_{1}, \ldots, e_{n}\right\}$ is the standard orthonormal basis in $R^{n}$. Therefore the polynomial $p$ is $\mathbf{H}-\mathbf{S S t a b l e . ~ T h u s ~ i t s ~ c o e f f i c i e n t s ~ a r e ~ s t r i c t l y ~}$ positive real numbers and all non-zero vectors $Y \in R_{+}^{n}$ belong to its open hyperbolic cone $C_{e}(p)$.

2. First, if $p \in \operatorname{Hom}_{+}(n, n)$ is $\mathbf{H}-\mathbf{S t a b l e}$ then clearly the scaled polynomial $p_{\mathbf{c}}$ is also H-Stable.

It follows from the definition of the Capacity that $\operatorname{Cap}\left(p_{\mathbf{c}}\right)=c_{1} \cdots c_{n} \operatorname{Cap}(p)$.

We get, by a direct computation, that

$$
\frac{\partial^{n}}{\partial x_{1} \ldots \partial x_{n}} p_{\mathbf{c}}(0, \ldots, 0)=c_{1} \cdots c_{n} \frac{\partial^{n}}{\partial x_{1} \ldots \partial x_{n}} p(0, \ldots, 0) \text {. }
$$


This proves that the set of $\mathbf{H}$-Stable extremal polynomials is invariant with respect to the scaling.

We need the following simple result (it was essentially proved in Lemma 3.8 from [8]).

Fact 5.9: Consider $p \in \operatorname{Hom}_{++}(n, n), p\left(x_{1}, \ldots x_{n}\right)=\sum_{r_{1}, \ldots, r_{n}} a_{r_{1}, \ldots, r_{n}} \prod_{1 \leq i \leq n} x_{i}^{r_{i}}$. Then there exists a positive vector $\mathbf{t}=:\left(t_{1}, \ldots, t_{n}\right) \in R_{++}^{n}, t_{1} \cdots t_{n}=1$ such that

$$
p\left(t_{1}, \ldots, t_{n}\right)=\operatorname{Cap}(p)=\inf _{x_{i}>0,1 \leq i \leq n ; x_{1} \cdots x_{n}=1} p\left(x_{1}, \ldots, x_{n}\right) .
$$

Consider the corresponding scaled polynomial $p_{\mathbf{t}}$. Then the polynomial $q=\frac{p_{\mathbf{t}}}{p_{\mathbf{t}}(1, \ldots, 1)}$ is doubly-stochastic. I.e. $\frac{\partial}{\partial x_{i}} q(1,1, \ldots, 1)=1,1 \leq i \leq n$..

Proof: Consider a subset

$$
T=\left\{\left(x_{1}, \ldots, x_{n}\right) \in R_{++}^{n}: x_{1} \cdots x_{n}=1 \quad \text { and } \quad p\left(x_{1}, \ldots, x_{n}\right) \leq p(1, \ldots, 1)\right\} .
$$

In order to prove that the infimum is attained, it is sufficient to show that the subset $T$ is compact. Clearly, $T$ is closed, and we need to prove that $T$ is bounded. Let $\left(x_{1}, \ldots, x_{n}\right) \in$ $T$ and assume WLOG that $\max _{1 \leq i \leq n} x_{i}=x_{1}$. Then

$$
a_{n, 0, \ldots, 0} x_{1}^{n} \leq p\left(x_{1}, \ldots, x_{n}\right) \leq p(1, \ldots, 1) \Rightarrow \max _{1 \leq i \leq n} x_{i} \leq \frac{p(1, \ldots, 1)}{a_{n, 0, \ldots, 0}}<\infty .
$$

This shows the desired boundness of $T$ and the existence of the minimum.

Consider a positive vector $\left(t_{1}, \ldots, t_{n}\right)$ such that $p\left(t_{1}, \ldots, t_{n}\right)=\operatorname{Cap}(p)$. Define $\alpha_{i}=$ $\log \left(t_{i}\right), 1 \leq i \leq n$. Then

$$
p\left(\exp \left(\alpha_{1}\right), \ldots, \exp \left(\alpha_{n}\right)\right)=\min _{\beta_{1}+\ldots \beta_{n}=0} p\left(\exp \left(\beta_{1}\right), \ldots, \exp \left(\beta_{n}\right)\right) .
$$

Therefore there exists the Lagrange multiplier $\gamma$ such that

$$
\frac{\partial}{\partial \alpha_{i}} p\left(\exp \left(\alpha_{1}\right), \ldots, \exp \left(\alpha_{n}\right)\right)=t_{i} \frac{\partial}{\partial t_{i}} p\left(t_{1}, \ldots, t_{n}\right)=\gamma, 1 \leq i \leq n .
$$

It follows from the Euler's identity that $\gamma=p\left(t_{1}, \ldots, t_{n}\right)$ and

$$
\frac{\partial}{\partial x_{i}} q(1, \ldots, 1)=\left(p\left(t_{1}, \ldots, t_{n}\right)\right)^{-1} t_{i} \frac{\partial}{\partial t_{i}} p\left(t_{1}, \ldots, t_{n}\right)=1
$$

Remark 5.10: It is easy to prove that, in fact, the minimum in (42) is attained uniquely. It was proved in [12] that if $p \in \operatorname{Hom}_{+}(n, n)$ is $\mathbf{H - S t a b l e ~ t h e n ~ t h e ~ m i n i m u m ~ i n ~ ( 4 2 ) ~ e x i s t s ~}$ and attained uniquely iff

$$
\frac{\partial^{n}}{\partial x_{i} \partial x_{j} \prod_{m \neq(i, j)} \partial x_{m}} q(0, \ldots, 0)>0: 1 \leq i \neq j \leq n .
$$




\section{Proof: (Proof of Theorem (5.7).)}

It follows from Proposition(5.8) and Fact(5.9) that we can assume without loss of generality that the $\mathbf{H}$-Stable extremal polynomial $p \in \operatorname{Hom}_{++}(n, n)$ is doubly-stochastic and all its coefficients are positive real numbers.

\section{Using uniqueness part of Lemma(3.2)}

Let $\left\{e_{1}, \ldots, e_{n}\right\}$ be the standard basis in $R^{n}$ and $p$ is now a H-SStable doublystochastic polynomial with positive coefficients, $p$ satisfies the equality (41). We need to look at the case of equality in (35). Recall the polynomial $q_{n-1}$ is given by $q_{n-1}\left(x_{1}, \ldots, x_{n-1}\right)=\frac{\partial}{\partial x_{n}} p\left(x_{1}, \ldots, x_{n-1}, 0\right)$.

Clearly, the polynomial $q_{n-1} \in \operatorname{Hom}_{++}(n-1, n-1)$ also has positive coefficients. Let

$$
q_{n-1}\left(t_{1, n}, \ldots, t_{n-1, n}\right)=\min _{x_{1}, \ldots, x_{n-1}>0, \prod_{1 \leq i \leq n-1} x_{i}=1} q_{n-1}\left(x_{1}, \ldots, x_{n-1}\right) .
$$

The existence of such a vector was proved in Proposition (5.9).

It follows from the uniqueness part of Lemma(3.2) that the univariate polynomial $R(t)=p\left(t_{1, n}, \ldots, t_{n-1, n}, t\right)=p\left(\sum_{1<i<n-1} t_{i, n-1} e_{i}+t e_{n}\right)$ has $n$ equal negative roots: $R(t)=b\left(t+a_{n}\right)^{n} ; a_{n}, b>0$. This fact implies, as in the second part of Example (5.5), that

$$
K_{n}=: e_{n}-\sum_{j \neq n} a_{j, n} e_{j} \in N u l l_{p}, a_{j, n}=\frac{t_{j, n}}{a_{n}}>0 .
$$

Since $p$ is doubly-stochastic, hence it follows from (39) that the coordinates of $K_{n}$ sum to zero. Which gives that $\sum_{j \neq n} a_{j, n}=1$.

In the same way, we get that there exists an $n \times n$ column stochastic matrix $A$ with the zero diagonal and the positive off-diagonal part such that the vectors

$$
K_{i}=: e_{i}-\sum_{j \neq i} a_{j, i} e_{j} \in N u l_{p}, i \leq i \leq n .
$$

2. Recall that our goal is to prove that $\operatorname{dim}\left(N u l_{p}\right)=n-1$. It follows that

$$
\operatorname{dim}\left(\operatorname{Null}_{p}\right) \geq \operatorname{dim}\left(L\left(K_{1}, \ldots, K_{n}\right)\right)=\operatorname{Rank}(I-A),
$$

where $L\left(K_{1}, \ldots, K_{n}\right)$ is the minimal linear subspace containing the set $\left\{K_{1}, \ldots, K_{n}\right\}$. Since the polynomial $p$ is non-zero thus $\operatorname{dim}\left(N u l_{p}\right) \leq n-1$. It is easy to see that $\operatorname{Rank}(I-A)=n-1$. Indeed, any principal $n-1 \times n-1$ submatrix of $I-A$ is strictly diagonally dominant and, therefore, is nonsingular. We finally conclude that $\operatorname{dim}\left(N u l l_{p}\right)=n-1$. 


\section{Comments}

1. Falikman [5] and Egorychev [4] publications were followed by a flurry of expository papers, which clarified and popularized the proofs. The author learned the Egorychev's proof from [17]. It is our guess that many scientists first learned about Alexandrov inequalities for mixed discriminants and Alexandrov-Fenchel inequalities for mixed volumes [1] in one of those expository papers. We would like to distinguish the following two papers: [16] and [25]. They both explicitly connected Alexandrov inequalities for mixed discriminants with homogeneous hyperbolic polynomials. The paper [16] was, essentially a rediscovery of Garding's theory [6]. Still, as the author had read [16] before reading [6], the paper [16] gave us the first hint for the possibility of our approach.

The paper [25], apparently written as a technical report in 1981 and published only in 2006 in an obscure book, is technically very similar to [16]. Besides, it implicitly introduced the Bapat's conjecture.

Other related publications are D. London's (univariate) papers [21],[20],[19].

As far as we know, there were no previously published connections between ShrijverValiant conjecture, which was thought to be of purely combinatorial nature, and stable/hyperbolic polynomials.

2. Two main ingredients of our approach, which make the proofs simple, are the usage of the notion of Capacity and Lemma (3.2). They together allowed the simple induction. The induction, used in this paper, is by partial differentiation. It is very similar to the inductive proofs of hyperbolic polynomials analogues of Alexandrov inequalities for mixed discriminants in [16],[25]. Using our terminology, these analogues correspond to the fact that the polynomial $q_{2}\left(x_{1}, x_{2}\right)=$ $\frac{\partial^{n-2}}{\partial x_{3} \ldots \partial x_{n}} p\left(x_{1}, x_{2}, 0, \ldots, 0\right)$ is either zero or $\mathbf{H}$-Stable provided the polynomial $p$ is H-Stable.

The idea to use Capacity in the context of permanents is implicit in [22]. The notion of Capacity was crucial for algorithmic results in [7], [8] as $\log (\operatorname{Cap}(p))=$ $\inf _{y_{1}+\cdots+y_{n}=0} \log \left(p\left(e^{y_{1}}, \ldots, e^{y_{n}}\right)\right)$ and the functional $\log \left(p\left(e^{y_{1}}, \ldots, e^{y_{n}}\right)\right)$ is convex for any polynomial with non-negative coefficients.

Probably, the papers [7], [8] were the first to reformulate Van der Waerden/Bapat conjectures as in inequality (27). Although quite simple, it happened to be a very enlighting observation.

3. Our, inductive by the partial differentiation, approach was initiated in [10]. The main tool there was Vinnikov-Dubrovin determinantal representation [29] of hyperbolic homogeneous polynomials in 3 variables. The paper [10] proved the implication $\operatorname{Cap}(p)>0 \Longrightarrow \frac{\partial^{n}}{\partial x_{1} \ldots \partial x_{n}} p(0, \ldots, 0)>0$ for H-Stable polynomials $p \in \operatorname{Hom}_{+}(n, n)$. Additionally, it was proved that in this H-Stable case the functional $\operatorname{Rank}_{p}(S)=$ 
$\max _{a_{r_{1}, \ldots, r_{n}} \neq 0} \sum_{j \in S} r_{j}$ is submodular and

$$
a_{r_{1}, \ldots, r_{n}}>0 \Longleftrightarrow \sum_{j \in S} r_{j} \leq \operatorname{Rank}_{p}(S): S \subset\{1, \ldots, n\} .
$$

The characterization (43) is a far reaching generalization of the Hall-Rado theorem. The paper [12] provides algorithmic applications of these results: strongly polynomial deterministic algorithms for the membership problem as for the support as well for the Newton polytope of $\mathbf{H}$-Stable polynomials $p \in \mathrm{Hom}_{+}(m, n)$, given as oracles.

\section{Acknowledgements}

The author is indebted to the anonymous reviewer for a very careful and thoughtful reading of the original version of this paper. Her/his numerous corrections and suggestions are reflected in the current version.

I would like to thank the U.S. DOE for financial support through Los Alamos National Laboratory's LDRD program.

\section{References}

[1] A. Aleksandrov, On the theory of mixed volumes of convex bodies, IV, Mixed discriminants and mixed volumes (in Russian), Mat. Sb. (N.S.) 3 (1938), 227-251.

[2] R. B. Bapat, Mixed discriminants of positive semidefinite matrices, Linear Algebra and its Applications 126, 107-124, 1989.

[3] Y.-B. Choe, J.G. Oxley, A. D. Sokal and D.G. Wagner, Homogeneous mltivariate polynomials with the half plane property, Advances in Applied Mathematics 32 (2004), 88- 187.

[4] G.P. Egorychev, The solution of van der Waerden's problem for permanents, Advances in Math., 42, 299-305, 1981.

[5] D. I. Falikman, Proof of the van der Waerden's conjecture on the permanent of a doubly stochastic matrix, Mat. Zametki 29, 6: 931-938, 957, 1981, (in Russian).

[6] L.Garding, An inequality for hyperbolic polynomials, Jour. of Math. and Mech., 8(6): 957-965, 1959.

[7] L.Gurvits and A. Samorodnitsky, A deterministic polynomial-time algorithm for approximating mixed discriminant and mixed volume, Proc. 32 ACM Symp. on Theory of Computing (Stoc-2000), ACM, New York, 2000.

[8] L.Gurvits and A. Samorodnitsky, A deterministic algorithm approximating the mixed discriminant and mixed volume, and a combinatorial corollary, Discrete Comput. Geom. 27: 531 -550, 2002. 
[9] L. Gurvits. Combinatorics hidden in hyperbolic polynomials and related topics, preprint (2004), available at http://xxx.lanl.gov/abs/math.CO/0402088.

[10] L. Gurvits, Combinatorial and algorithmic aspects of hyperbolic polynomials, 2004; available at http://xxx.lanl.gov/abs/math.CO/0404474.

[11] L. Gurvits, A proof of hyperbolic van der Waerden conjecture: the right generalization is the ultimate simplification, Electronic Colloquium on Computational Complexity (ECCC)(103): (2005) and arXiv:math/0504397.

[12] L. Gurvits, Hyperbolic polynomials approach to Van der Waerden/Schrijver-Valiant like conjectures: sharper bounds, simpler proofs and algorithmic applications, Proc. 38 ACM Symp. on Theory of Computing (StOC-2006),417-426, ACM, New York, 2006.

[13] L. Gurvits, Van der Waerden Conjecture for Mixed Discriminants, Advances in Mathematics, 2006.

[14] L. Hormander, Analysis of Linear Partial Differential Operators, Springer-Verlag, New York, Berlin, 1983.

[15] V. L. Kharitonov and J. A. Torres Munoz, Robust Stability of Multivariate Polynomials. Part 1: Small Coefficients Pertubrations, Multideminsional Systems and Signal Processing, 10 (1999), 7-20.

[16] A.G. Khovanskii, Analogues of the Aleksandrov-Fenchel inequalities for hyperbolic forms, Soviet Math. Dokl. 29(1984), 710-713.

[17] Knuth, Donald E. A permanent inequality. Amer. Math. Monthly 88 (1981), no. 10, 731-740, 798.

[18] N. Linial, A. Samorodnitsky and A. Wigderson, A deterministic strongly polynomial algorithm for matrix scaling and approximate permanents, Proc. 30 ACM Symp. on Theory of Computing, ACM, New York, 1998.

[19] D. London, On the van der Waerden conjecture and zeros of polynomials. Linear Algebra Appl. 45 (1982), 35-41.

[20] D. London, On the van der Waerden conjecture for matrices of rank two. Linear and Multilinear Algebra 8 (1979/80), no. 4, 281-289

[21] D. London, On a connection between the permanent function and polynomials. Linear and Multilinear Algebra 1 (1973), 231-240.

[22] D. London, On matrices with a doubly stochastic pattern. J. Math. Anal. Appl. 34 $1971648-652$.

[23] H.Minc, Permanents, Addison-Wesley, Reading, MA, 1978.

[24] James Renegar, Hyperbolic Programs, and Their Derivative Relaxations, Foundations of Computational Mathematics 6(1): 59-79 (2006)

[25] J. Peetre, Van der Waerden Conjecture and Hyperbolicity, in Kaljulaid, Uno; Semigroups and automata. Selecta Uno Kaljulaid (1941-1999). Edited by Jaak Peetre and Jaan Penjam, pp. 225-232, IOS Press, Amsterdam, 2006. 
[26] A. Schrijver and W.G.Valiant, On lower bounds for permanents, Indagationes Mathematicae 42 (1980) 425-427

[27] A. Schrijver, Counting 1-factors in regular bipartite graphs, Journal of Combinatorial Theory, Series B 72 (1998) 122-135.

[28] M. Voorhoeve, A lower bound for the permanents of certain $(0,1)$ matrices, Indagationes Mathematicae 41 (1979) 83-86.

[29] V. Vinnikov, Selfadjoint determinantal representations of real plane curves. Math. Ann. 296 (1993), 453-479.

[30] I. M. Wanless, Addendum to Schrijver's work on minimum permanents, Combinatorica 26 (6) (2006) 743-745. 


\section{Corrigendum - submitted May 29, 2008}

1. On page 5 , the formula in the 4 th item of Definition 2.1

$$
p\left(x_{1}, \ldots, x_{n}\right)=\sum_{r_{1}+\cdots+r_{n}=1} a_{r_{1}, \ldots, r_{n}} \prod_{1 \leq i \leq n} x_{i}^{r_{i}} .
$$

should be replaced by

$$
p\left(x_{1}, \ldots, x_{n}\right)=\sum_{r_{1}+\cdots+r_{n}=n} a_{r_{1}, \ldots, r_{n}} \prod_{1 \leq i \leq n} x_{i}^{r_{i}} .
$$

2. The formula (9) on page 5 should be replaced by

$$
\sum_{r_{1}+\cdots+r_{n}=n} a_{r_{1}, \ldots, r_{n}} r_{j}=1: 1 \leq j \leq n .
$$

3. The formula (10) on page 6 should be replaced by

$$
\sum_{r_{1}+\cdots+r_{n}=n} a_{r_{1}, \ldots, r_{n}}=1
$$

4. The second formula on page 6 ,

$$
\log \left(p\left(x_{1}, \ldots, x_{n}\right)\right) \geq \sum_{r_{1}+\cdots+r_{n}=1} a_{r_{1}, \ldots, r_{n}} r_{i} \log \left(x_{i}\right)=\log \left(x_{1} \cdots x_{n}\right) .
$$

should be replaced by

$$
\log \left(p\left(x_{1}, \ldots, x_{n}\right)\right) \geq \sum_{r_{1}+\cdots+r_{n}=n} a_{r_{1}, \ldots, r_{n}} \sum_{1 \leq i \leq n} r_{i} \log \left(x_{i}\right)=\log \left(x_{1} \cdots x_{n}\right) .
$$

All these corrections deal with the same typo : $r_{1}+\cdots+r_{n}=1$ is replaced by $r_{1}+\cdots+r_{n}=n$

5. The last formula in Remark 5.10 on page 20 ,

$$
\frac{\partial^{n}}{\partial x_{i} \partial x_{j} \prod_{m \neq(i, j)} \partial x_{m}} q(0, \ldots, 0)>0: 1 \leq i \neq j \leq n .
$$

should be replaced by

$$
\frac{\partial^{n}}{\left(\partial x_{i}\right)^{2} \prod_{k \notin\{i, j\}} \partial x_{k}} p(0, \ldots, 0)>0, \quad \frac{\partial^{n}}{\left(\partial x_{j}\right)^{2} \prod_{k \notin\{i, j\}} \partial x_{k}} p(0, \ldots, 0)>0: 1 \leq i \neq j \leq n .
$$

